\title{
IDENTIFYING THE APE BEAT IN THE WILD: RHYTHMIC INDIVIDUAL SIGNATURES FROM THE SOUNDS OF MANUAL FRUIT CRACKING IN FONGOLI CHIMPANZEES
}

\author{
ADRIEN MEGUERDITCHIAN ${ }^{* 1}$, ADELYNE VUILLEMIN ${ }^{1}$, and JILL D. PRUETZ ${ }^{2}$ \\ *Corresponding Author: adrien.meguerditchian@univ-amu.fr \\ ${ }^{1}$ Laboratoire de Psychologie Cognitive, Brain and Language Research Institute, Aix- \\ Marseille Univ, CNRS, Marseille, France \\ ${ }^{2}$ Department of Anthropology, Texas State University, San Marcos, TX, USA
}

Rhythm is one of the key components of the temporal structure of language at both production and perception levels. To investigate the phylogenetical origins of this property, comparative approach on the rhythmic properties of vocal communication, acoustic or motor behaviors has been developed between human and a range of animal species including birds, sea lions and nonhuman primates (e.g. Ravignani et al., 2016; Dufour et al., 2015; Cook et al., 2013). In great apes specifically, manual beating - "drumming" - on a tree, object or body part has been documented in different apes' species and contexts including display, play or travels (e.g. Arcadi et al., 1998). "Drumming" in great apes has been considered as a behavior of interest for investigating the ancestral prerequisites to rhythmic processing in humans (Dufour et al., 2015; Fitch 2006). One can question what functional advantage such rhythmic properties may provide to favor the selection of its underlying processing capacity across evolution from our common ancestor. Based on preliminary evidence for individual distinctiveness of drumming acoustic patterns in male chimpanzees, it has been proposed that these rhythmic cues may help the chimpanzees to recognize unseen conspecifics by their drumming behaviors (e.g. Arcadi et al., 1998). Although poorly studied and unclear, the question of the individual distinctiveness of rhythmic acoustic behaviors in animals remains essential to investigate this latter hypothesis.

In the present study conducted in a group of habituated wild chimpanzees living on the field site of Fongoli, South-East Senegal (Pruetz \& Bertolani, 2007), we 
investigated the individual rhythmic acoustic patterns generated by 4 females and 10 males when cracking a baobab fruit (Strydinos spp). In holding the fruit by its stem, this unimanual behavior consists of repetitively slapping the fruit on a hard surface (the ground, baobab tree's branch or root) in order to make its shell softer before opening and eating it. It resulted in bouts of long-distance rhythmic sounds which were recorded on site. Each bout of baobab fruit cracking was further analyzed to extract the individual inter-beat durations within the bout. Among the 151 bouts collected from 40 hours of video clips, we identified 3 types of rhythmic patterns produced by the chimpanzees: (1) Repetitive power beats in 8 subjets; (2) Repetitive sequences of 1 power beat + followed by either 1 rebound soft beat (in 9 subjects) or (3) + by 2 consecutive soft rebound beats (in 5 subjects). In addition, for each rhythmic patterns, the analyses of the individual averaged inter-beat durations across subjects showed significant differences between individuals.

These findings clearly indicate that cracking baobab fruit indirectly generated distinct inter-beat individual signatures in most of the chimpanzees. It is then not excluded that these rhythmic cues may help chimpanzees to recognize unseen conspecifics. Given the functional advantages of such potential long distance individual identifications, it constitutes an ideal candidate for selective pressure in favor of rhythmic processing prerequisites across primate evolution.

\section{Acknowledgements}

We are very grateful to William D. Hopkins, Dondo Kanté, Tama Sadiakhou, Wally \& Syra Camara. Grants: Fondation Fyssen

\section{References}

Arcadi, A. C., Robert, D. \& Boesch, C. (1998). Buttress drumming by wild chimpanzees: temporal patterning, phrase integration into loud calls and preliminary evidence for individual distinctiveness. Primates, 39, 505-518.

Cook, P., Rouse, A., Wilson, M. \& Reichmuth, C. A. (2013). California sea lion (Zalophus californianus) can keep the beat: motor entrainment to rhythmic auditory stimuli in a non vocal mimic. Journal of Comparative Psychology, 127, 412-427.

Fitch, W. T. (2006). The biology and evolution of music: A comparative perspective. Cognition, 100, 173-215.

Pruetz, J. D., \& Bertolani, P. (2007), Savanna Chimpanzees, Pan troglodytes verus, Hunt with Tools. Current Biology 17, 412-417.

Ravignani, A. et al. (2016) 'What Pinnipeds Have to Say about Human Speech, Music, and the Evolution of Rhythm', Frontiers in Neuroscience, 10, 274. 